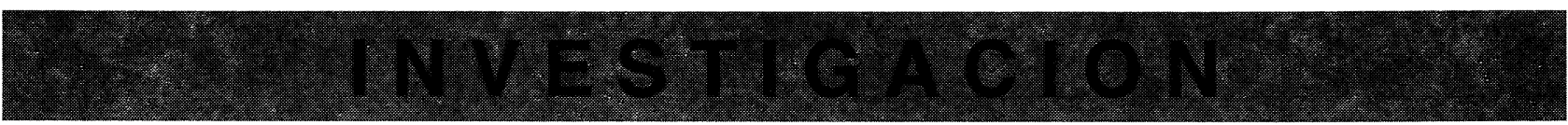

\title{
Composición en ácidos grasos de los aceites de semillas de especies mediterráneas
}

\author{
Por E. Ucciani (1), G. Mallet (1), J. Gamisans (2) y M. Gruber (2) \\ (1) Laboratoire de Chimie Organique Appliquée, CNRS URA 1409. \\ (2) Laboratoire de Botanique et Ecologie Méditerranéennes, CNRS URA 1152. \\ Faculté des Sciences. 13397 Marseille Cedex 20 - France
}

\section{RESUMEN}

Composición en ácidos grasos de los aceites de semillas de especies mediterráneas.

En el marco de investigaciones sobre los lípidos de las plantas superiores, se presenta la composición en ácidos grasos de once especies. Estas especies pertenecen a nueve familias diferentes y la mayoria son frecuentes en la flora mediterránea.

Numerosos aceites (Arctium tomentosum, Hypericum perforatum, Linaria vulgaris, Pulicaria dysenterica y Smilax aspera) contienen glicéridos parciales y ácidos grasos libres. El aceite de Arctium tomentosum, y en menor grado, el de Smilax aspera muestran una banda espectral trans en espectrometría IR. En los aceites de Pulicaria dysenterica y de Silybum marianum pequeñas cantidades de ceras han sido puestas de manifiesto.

Las composiciones en ácidos grasos se aproximan a las de los aceites alimenticios e industriales conocidos. Tres especies contienen un ácido graso útil a un nivel elevado: Alliaria petiolata ( $41,5 \%$ de ácido erúcico), Linaria vulgaris y Pulicaria dysenterica (respectivamente 70 y $72 \%$ de ácido linoléico). En el aceite de Arctium tomentosum cuatro ácidos grasos menores no han sido identificados: uno de ellos podría ser el octadeca-3(t), 9(c), 12(c) trienóico. Pero se alcanza el máximo de complejidad con el aceite de Atriplex hortensis: con treinta y tres ácidos grasos de los cuales quince son desconocidos.

PALABRAS-CLAVE: Aceite de semilla - Acido graso (composición) Flora mediterránea.

\section{SUMMARY}

Fatty acid composition of seed oils of Mediterranean herbaceous species.

In the context of research into the lipids of higher plant, the fatty acid composition of eleven species is reported. These species belong to nine families, and most of them are common in the Mediterranean flora.

Oils from some plants (Arctium tomentosum, Hypericum perforatum, Linaria vulgaris, Pulicaria dysenterica, Smilax aspera) were partially hydrolyzed, and contained free fatty acids. Arctium tomentosum seed oil and, to a lesser extent, Smilax aspera seed oil, showed a trans absorption band in their IR spectra. Small quantities of waxes were present in Pulicaria dysenterica and Sylibum marianum seed oil.

The fatty acid composition of the oils from these plants approaches levels found in food oils as well as industrial oils. Three species showed high levels of their predominant fatty acid: Alliaria petiolata (erucic acid, $41.5 \%$ ), Linaria vulgaris and Pulicaria dysenterica (linoleic acid, 70 and $72 \%$ respectively). In the seed oil of Arctium tomentosum four fatty acids were not identified: one of them could be $3(t), 9(\mathrm{c}), 12(\mathrm{c})$ octadecatrienoic acid. But the most complex composition was that of Atriplex hortensis oil, which contained thirty three fatty acids, of which fifteen were unknown.

KEY-WORDS: Fatty acid (composition) - Mediterranean flora - Seed oil.

\section{INTRODUCCION}

Interesados desde hace unos años por los antioxidantes fenólicos (Chevolleau, 1992 a,b - Cerrati, 1992) y por los lípidos de resenva (Ferlay, 1993), hemos encontrado en la flora mediterránea un campo de investigación aún poco explotado. Esta flora está globalmente adaptada a la sequedad. Las especies mediterráneas están aquí ampliamente representadas, pero las plantas eurosiberianas constituyen también un grupo importante.

Muy perturbado por el hombre desde hace milenios, el paisaje vegetal se caracteriza por la importancia de los cultivos -sin embargo en regresión- y de los medios abiertos (eriales, pastizales, zonas incendiadas). En estos medios perturbados, unas cuantas especies extranjeras, particularmente subtropicales, traídas voluntariamente o no por el hombre, se han naturalizado o son subespontáneas.

Las especies aquí estudiadas son representativas de los tres conjuntos citados :

- Acanthus mollis L. (Acanthaceae), especie mediterránea occidental cuya área natural resulta difícil delimitar, pues ha sido cultivada desde la antigüedad. Herbácea de gran tamaño, se naturaliza frecuentemente cerca de las viviendas (0-100 m).

- Alliaria petiolata (Bieb.) Cavara \& Grande (Brassicaceae), especie herbácea paleotemplada, bastante nitrófila, frecuente en los lugares húmedos $y$ frescos (0-1000 m).

- Arctium tomentosum Miller (Asteraceae), especie euroasiática templada, nitrófila, que se encuentra en setos y eriales (1000-1500 m).

- Atriplex hortensis L. (Chenopodiaceae), especie herbácea euroasiática, frecuente en los cultivos (0$600 \mathrm{~m}$ ).

- Hypericum perforatum L. (Clusiaceae), herbácea paleotemplada volviéndose subcosmopolita, muy frecuente en la región mediterránea en eriales y linderos silvestres de 0 a $1600 \mathrm{~m}$.

- Linaria vulgaris Miller (Scrophulariaceae), especie herbácea euroasiática presente en eriales y otros lugares antrópicos (0-1500 m). 
- Mirabilis jalapa L. (Nyctaginaceae), herbácea originaria de Perú, frecuentemente cultivada en jardines y subespontánea en eriales y zonas antrópicas $(0-600 \mathrm{~m})$.

- Pulicaria dysenterica Gaertner (Asteraceae), especie herbácea mediterránea, colonizando majales, prados húmedos y cunetas $(0-1500 \mathrm{~m})$.

- Rosa rubiginosa L. (Rosaceae), matorral euroasiático presente en la región mediterránea en setos, bosques abiertos y claros (100-1400 m).

- Silybum marianum (L.) Gaertner (Asteraceae), gran cardo mediterráneo, localmente abundante en eriales y vertederos (0-1100 $\mathrm{m}$ ).

- Smilax aspera L. (Liliaceae), bejuco espinescente paleosubtropical, muy frecuente en garrigas y matorrales mediterráneos $(0-1000 \mathrm{~m})$.

\section{MATERIAL Y METODOS}

\subsection{Extracción del aceite y análisis preliminares}

Las semillas son escogidas y lavadas rápidamente con agua y luego con etanol, antes de ser secadas bajo vacio (3-5 $\mathrm{mm} \mathrm{Hg}$ ) a $40^{\circ} \mathrm{C}$, en la oscuridad.

La trituración se hace en un triturador de palas IKA Labortechnik $A 10$ a $20^{\circ} \mathrm{C}$. La extracción se realiza en un aparato de Soxhlet, con hexano durante $6 \mathrm{~h}$. El contenido en aceite de las semillas es la media de dos medidas.

Los aceites se analizan por cromatografía en capa fina (CCF) de Kieselgel 60 F 254 (Merck) con hexano 80 : éter 20 (en volumen) como solvente de desarrollo. Las placas son reveladas por pulverización con ácido sulfúrico concentrado en éter (al $3 \%$ peso : volumen) seguida de calentamiento durante unos segundos a $230-259^{\circ} \mathrm{C}$. Los espectros en infrarrojo (IR) están registrados en un aparato Beckman Acculab IV en cuba sellada de $0,5 \mathrm{~mm}$ de espesor, estando los aceites en solución en tetracloruro de carbono (al $2 \%$ peso : volumen). Se hacen dos reacciones coloreadas: la prueba de Halphen en solución (Wolff, 1968) y el revelado con ácido pícrico en CCF (Fioriti, 1968).

\subsection{Preparación de los ésteres metílicos.}

Los aceites se saponifican con potasa etanólica N ( 5 $\mathrm{ml} / \mathrm{g}$ de aceite) y el insaponificable se extrae según la Norma 2.401 (IUPAC, 1979) substituyendo el éter por el metil-t.butil éter (MTBE). La fase hidroalcohólica que contiene los jabones se diluye luego en una solución saturada de $\mathrm{NaCl}$, y se acidifica con $\mathrm{HCl} 4 \mathrm{~N}$. Los ácidos grasos se extraen por el MTBE. Después de un lavado a neutralidad y evaporación del disolvente, los ácidos grasos son recogidos por metanol clorhídrico (5 $\mathrm{ml}$ por $\mathrm{g}$ de ácido graso). El metanol clorhídrico se prepara añadiendo $120 \mathrm{ml}$ de cloruro de acetilo (Prolabo) a $880 \mathrm{ml}$ de metanol anhidro.

Después de dos horas de ebullición, la solución es enfriada y diluida con agua. Los ésteres son recogidos en hexano $(50 \mathrm{ml} / \mathrm{g})$. La fase orgánica se lava con agua a neutralidad, luego con carbonato de potasio acuoso al 5\% (peso: vol.) y luego con agua. Después de secarse sobre sulfato de sodio anhidro, la solución de ésteres metílicos en hexano está lista para la cromatografía gaslíquido (CGL).

\subsection{Análisis por cromatografía gas-líquido.}

Se utiliza un aparato Girdel 300. Comprende un inyector-divisor y un detector de ionización de llama. Los tiempos de retención y las superficies de los picos se miden con un integrador Enica 21. Se utilizan dos columnas capilares de silice fundida:

- para el análisis rápido, OV $17(\mathrm{~L}=15 \mathrm{~m}$, diam.int. = $0,25 \mathrm{~mm}$, espesor de película $=0,2 \mu \mathrm{m}$ ), temperatura inyector $320^{\circ} \mathrm{C}$, detector $300^{\circ} \mathrm{C}$, horno $150-300^{\circ} \mathrm{C}$ $\left(10^{\circ} \mathrm{C} / \mathrm{min}\right.$.), gas portador Helio 0,8 bar.

- para el análisis en alta resolución, Carbowax 20M $(\mathrm{L}=30 \mathrm{~m}$, diam.int. $=0,3 \mathrm{~mm}$, espesor película $=$ $0,25 \mu \mathrm{m}$ ), temperatura inyector $240^{\circ} \mathrm{C}$, detector $250^{\circ} \mathrm{C}$, horno $185^{\circ} \mathrm{C}$, Helio 0,7 bar.

\section{RESULTADOS Y DISCUSION}

Las especies estudiadas son presentadas en la TABLA $\mathrm{I}$, que pone de relieve el contenido en aceite y en insaponificable, así como la presencia eventual de constituyentes no-glicerídicos. El contenido en aceite de las semillas varía en límites amplios, del 2,6 al 27,2\%. En líneas generales estas especies son pobres en aceite. La proporción de insaponificable varía del 0,8 a $8,7 \%$. Tres especies se distinguen en cuanto a su contenido elevado en insaponificable : A.mollis (8,7\%), H.perforatum $(7,3 \%)$ y M.jalapa $(3,7 \%)$. Con mas o menos del $2 \%$ de insaponificable respecto a la semilla, H.perforatum aparece como una especie interesante para aplicaciones eventuales cosméticas.

Cinco aceites presentan acidez libre. Estando las semillas en buen estado, la acidez notada sería pues la señal de una actividad lipásica ocurrida antes o durante el secado. Estos ácidos grasos libres son revelados por CCF $\left(\mathrm{R}_{\mathrm{F}}=\right.$ $0,15)$. Además, se encuentran ceras en P.dysenterica y S.marianum, caracterizadas por $\operatorname{CCF}\left(R_{F}=0,90\right)$. Las ceras, son ésteres de ácidos grasos y alcoholes grasos de cadena larga, encontradas a menudo en los aceites de las Asteraceae (Hamilton, 1972). Así, ácidos grasos libres y ceras son los únicos compuestos no glicerídicos identificados. Los aceites estudiados no contienen ni estólidos o poliésteres $\left(R_{F}=0,80\right)$ (Madrigal et al.,1982), ni cianolípidos (IR $2220 \mathrm{~cm}^{-1}, R_{F}=0,65$ ) (Micolajczak, 1977).

Los ácidos grasos inhabituales se pueden poner de relieve por IR así como por reacciones coloreadas. La ausencia de ácidos grasos ciclopropénicos es confirmada en todos los aceites estudiados, por el hecho de su respuesta negativa a la prueba de Halphen (Wolff, 1968). En cambio, gracias a la reacción de los epóxidos con el ácido pícrico sobre placa de CCF (Fioriti, 1968), se pueden revelar vestigios de epóxidos 
en el aceite de S.marianum. Pero la débil intensidad de la coloración puede hacer pensar en la reacción de productos de auto-oxidación. Los espectros IR no contienen bandas características de los alcoholes $(\mathrm{v}(\mathrm{OH})$ asociados 3200$3300 \mathrm{~cm}^{-1}$ ) lo que tiende a demostrar la ausencia de ácidos grasos hidroxilados. En cambio se revela una banda trans $\left(\gamma(\mathrm{CH})\right.$ muy débil en $S$. aspera a $965 \mathrm{~cm}^{-1}$, y mas evidente en A.tomentosum a $972 \mathrm{~cm}^{-1}$ ). Estas bandas pueden ser atribuidas a productos de auto-oxidación en el caso de S.aspera, y a un constituyente nuevo en el caso de A.tomentosum, que no puede ser el ácido elaídico, ya que éste absorbe a $965-967 \mathrm{~cm}^{-1}$.
En conclusión, estas pruebas preliminares son necesarias en el caso de aceites desconocidos. Permiten revelar la presencia de constituyentes inhabituales, aunque sea en pequeñas cantidades, como es el caso de los aceites estudiados.

Los ésteres metílicos, obtenidos por esterificación de los ácidos grasos, después de la extracción del insaponificable, se analizan por cromatografía en fase gaseosa. En análisis rápido el elemento mas pesado encontrado es el nervonato de metilo, 24:1 $\Delta 15$, caracterizado por comparación con el lignocerato de metilo, 24:0. Este último elemento se encuentra en cantidades leves en varios aceites.

Tabla I

Características generales de los aceites de semillas de diversas plantas de la flora mediterránea

\begin{tabular}{|c|c|c|c|}
\hline especie & $\begin{array}{l}\% \text { aceite } / \\
\text { semilla }\end{array}$ & $\begin{array}{c}\% \text { insaponificable / } \\
\text { aceite }\end{array}$ & $\begin{array}{l}\text { constituyentes } \\
\text { atípicos (*) }\end{array}$ \\
\hline $\begin{array}{l}\text { Acanthus mollis } \\
\text { (Acanthaceae) }\end{array}$ & 2,6 & 8,7 & - \\
\hline $\begin{array}{c}\text { Alliaria petiolata } \\
\text { (Brassicaceae) }\end{array}$ & 15,4 & 2,1 & - \\
\hline $\begin{array}{l}\text { Arctium tomentosum } \\
\text { (Asteraceae) }\end{array}$ & 11,6 & 1,6 & $\begin{array}{l}\text { ácidos grasos } \\
\text { libres +, trans ++ }\end{array}$ \\
\hline $\begin{array}{l}\text { Atriplex hortensis } \\
\text { (Chenopodiaceae) }\end{array}$ & 7,5 & 2,7 & - \\
\hline $\begin{array}{l}\text { Hypericum perforatum } \\
\text { (Clusiaseae) }\end{array}$ & 27,2 & 7,3 & $\begin{array}{c}\text { ácidos grasos } \\
\text { libres }+\end{array}$ \\
\hline $\begin{array}{l}\text { Linaria vulgaris } \\
\text { (Scrophulariaceae) }\end{array}$ & 7,2 & 1,9 & $\begin{array}{c}\text { ácidos grasos } \\
\text { libres }+\end{array}$ \\
\hline $\begin{array}{c}\text { Mirabilis jalapa } \\
\text { (Nyctaginaceae) }\end{array}$ & 4,2 & 3,7 & - \\
\hline $\begin{array}{c}\text { Pulicaria dysenterica } \\
\text { (Asteraceae) }\end{array}$ & 11,7 & 0,8 & $\begin{array}{l}\text { ceras +, ácidos } \\
\text { grasos libres + }\end{array}$ \\
\hline $\begin{array}{l}\text { Rosa rubiginosa } \\
\text { (Rosaceae) }\end{array}$ & 9,5 & 0,8 & - \\
\hline $\begin{array}{l}\text { Silybum marianum } \\
\text { (Asteraceae) }\end{array}$ & 24,2 & 1,1 & $\begin{array}{c}\text { ceras }+, \\
\text { epóxidos }+\end{array}$ \\
\hline $\begin{array}{l}\text { Smilax aspera } \\
\quad \text { (Liliaceae) }\end{array}$ & 7,4 & 2,2 & $\begin{array}{l}\text { ácidos grasos } \\
\text { libres }++, \text { trans }+\end{array}$ \\
\hline
\end{tabular}

$\left(^{\star}\right)+=$ proporción muy pequeña, $++=$ proporción pequeña

Para la identificación de los ésteres en análisis de alta resolución, hemos utilizado la Longitud Equivalente de Cadena (LEC) en isotermo, según la relación bien conocida (Tranchant, 1982) :

$$
\operatorname{LEC}_{(X)}=\frac{2\left[\log T_{R(X)}^{\prime}-\log T_{R(n)}^{\prime}\right]}{\log T_{R(n+2)}^{\prime}-\log T_{R(n)}^{\prime}}
$$

en donde $\mathrm{X}$ es el número de carbonos de un éster desconocido, $\mathrm{n}$ el número de carbonos de un éster saturado conocido y $n+2$ el número de carbonos de otro éster saturado con dos carbonos más, siendo $T_{R}$ el tiempo de retención reducido. Los valores obtenidos, reunidos en la TABLA II, se comparan a los de la literatura (Peyronel, 1984-Mallet, 1987). Se pueden apreciar algunas diferencias debidas esencialmente al modelo matemático escogido. Hemos elegido un modelo de primer orden, mientras que los modelos de la literatura son a menudo de segundo orden. 
Tabla II

Longitud de Cadena Equivalente (L.E.C.) de los ésteres metílicos de ácidos grasos

\begin{tabular}{|c|c|c|c|}
\hline $\begin{array}{l}\text { ésteres } \\
\text { metílicos }\end{array}$ & $\begin{array}{c}\text { este } \\
\text { trabajo }\end{array}$ & $\begin{array}{c}\text { LEC } \\
\text { ref. (a) }\end{array}$ & ref. (b) \\
\hline $12: 0$ & 12,00 & 11,96 & - \\
\hline $14: 0$ & 14,00 & 13,99 & - \\
\hline $16: 0$ & 16,00 & 15,99 & - \\
\hline $16: 1 \Delta 7$ & 16,18 & 16,20 & 16,14 \\
\hline $16: 1 \Delta 9$ & 16,26 & 16,26 & 16,29 \\
\hline $16: 2 \Delta 7,10$ & 16,62 & - & 16,62 \\
\hline $16: 2 \Delta 9,12$ & 16,86 & - & 16,86 \\
\hline $16: 3 \Delta 7,10,13$ & 17,50 & - & 17,47 \\
\hline $18: 0$ & 18,00 & 17,97 & - \\
\hline $18: 1 \Delta 9$ & 18,23 & 18,22 & 18,24 \\
\hline $18: 1 \Delta 11$ & 18,29 & 18,29 & 18,27 \\
\hline $18: 2 \Delta 9,12$ & 18,66 & 18,65 & 18,65 \\
\hline $18: 2 \Delta 11,14$ & 18,84 & - & 18,82 \\
\hline $18: 3 \Delta 6,9,12$ & 18,92 & 18,92 & 18,91 \\
\hline $18: 3 \Delta 9,12,15$ & 19,27 & 19,25 & 19,27 \\
\hline $18: 4 \Delta 6,9,12,15$ & 19,53 & 19,54 & 19,52 \\
\hline $20: 0$ & 20,00 & 20,01 & - \\
\hline $20: 1 \Delta 5$ & 20,07 & - & 20,07 \\
\hline $20: 1 \Delta 11$ & 20,19 & 20,16 & 20,17 \\
\hline $20: 1 \Delta 13$ & 20,24 & 20,26 & 20,26 \\
\hline $20: 2 \Delta 11,14$ & 20,60 & 20,62 & 20,61 \\
\hline $22: 0$ & 22,00 & 22,01 & - \\
\hline $22: 1 \Delta 13$ & 22,15 & 22,16 & 22,17 \\
\hline $22: 1 \Delta 15$ & 22,21 & - & - \\
\hline 24: 0 & 24,00 & 23,99 & - \\
\hline $24: 1 \Delta 15$ & 24,16 & 24,15 & 24,16 \\
\hline
\end{tabular}

(a) Peyronel et al., 1984

(b) Mallet, 1987

Siendo los picos en su mayoría identificados por su LEC, las composiciones de ácidos grasos de los aceites estudiados pueden entonces ser establecidas, como se indica en la TABLA III. El examen de los resultados muestra que, fuera de A.tomentosum y A.hortensis, las composiciones se aproximan a las de los aceites alimenticios e industriales conocidos:

- los ácidos inferiores (12:0 y 14:0) son poco frecuentes y poco abundantes, excepto en el caso de S.aspera.

- el ácido palmítico (16:0) se situa entre 3,2 y 19,1\% ; el ácido palmitoléico (16: $1 \Delta 9$ ) se presenta en pequeña cantidad en todos los aceites menos el de A.mollis en donde la proporción alcanza el 9,1\%. Otros ácidos en $\mathrm{C} 16$, poco frecuentes, son revelados, entre otros 16: $1 \Delta 7,16: 2 \Delta 7,10,16: 2 \Delta 9$, 12 y 16: $3 \Delta 7,10,13$. Su caracterización no es segura, pues los valores de LEC divergen sensiblemente. Por otra parte, estos ácidos están presentes a menudo en proporciones muy pequeñas. Hemos probado, sin éxito, caracterizarlos por espectrometría de masa acoplada a la CGL.

- el ácido esteárico (18:0) se encuentra a un nivel relativamente bajo en todos los aceites, cosa bastante habitual. Se identifican dos ácidos monoénicos, el oléico ( 18: $1 \Delta 9$ ) y el cis-vaccénico (18: $1 \Delta$ 11). El primero varía del 5,2 al $45,4 \%$. Es poco frecuente, en los aceites de semillas, que el ácido oléico baje por debajo del $10 \%$. En cuanto al ácido cis-vaccenico, es excepcional que exceda de las proporciones aquí indicadas; es un ácido graso más frecuente en los lípidos de pulpa (Mallet, 1988).

El ácido linoléico $(18: 2 \Delta, 9,12)$ es más abundante que el ácido oléico en nueve aceites sobre once. La concentración varía del 13,1 al $72,2 \%$. Dos especies -L.vulgaris y P.dysenterica- muestran una composición en ácido linoléico comparable a la del aceite de pepitas de uva y superior a la del aceite de girasol. En algunos casos -A.hortensis, M.jalapa, P.dysenterica- se encuentra otro ácido diénico, el isómero $\Delta 11,14$, en cantidad siempre reducida.

El ácido linolénico $(18: 3 \Delta 9,12,15)$ poco abundante en los aceites alimenticios, es uno de los componentes importantes de los aceites de H.perforatum, R.rubiginosa, A. tomentosum, M.jalapa y A.hortensis. Se nota, en cambio, la ausencia de ácido gammalinolénico $(18: 3 \Delta 6,9,12)$ del cual la $L E C=18,92$ permite una identificación sin ambigüedad.

- Los elementos en C20 -aráquico (20:0), gondoico (20:1 $\Delta 11)$, eicosadienóico $(20: 2 \Delta 11,14)$ - presentan solo un interés limitado, no sobrepasando su proporción el 5 por ciento.

- El ácido behénico (22:0) nunca es muy abundante, en cambio el ácido erúcico (22:1 $\Delta 13)$, constituyente principal de los aceites de Brassicaceae, alcanza el nivel del $41,4 \%$ en el aceite de A.petiolata. El ácido erúcico es a menudo acompañado de su homólogo superior, el ácido nervónico (24:1 $\Delta 15)$, presente únicamente en las Brassicaceae, mientras que el ácido lignocérico (24:0) se encuentra en pequeña cantidad en los lípidos de numerosas familias botánicas.

Un cierto número de ácidos grasos menores figuran en las composiciones dadas en la TABLA III. Fuera de los ácidos en $\mathrm{C} 12, \mathrm{C} 14$ y $\mathrm{C} 16$ ya citados, se encuentran ácidos cuya LEC está comprendida entre 16,00 y 18,00 , que no han podido ser identificados : uno en A.tomentosum $(0,1 \%)$, siete en $A$.hortensis (1,3\% en total) y uno en P.dysenterica $(0,7 \%)$. 
Tabla III

Composición en ácidos grasos de los aceites de semillas de diversas plantas de la flora mediterránea.

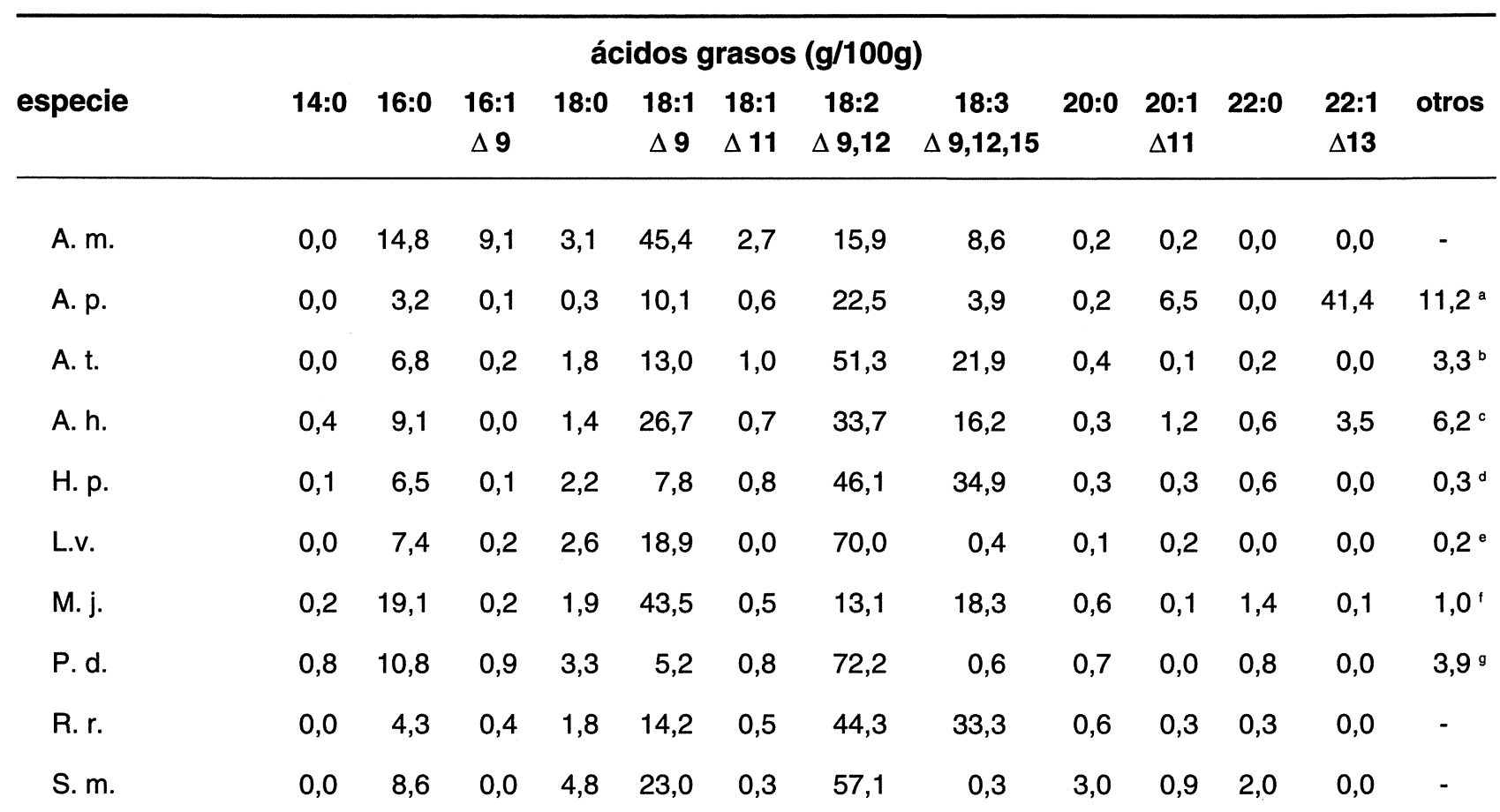

(a): $16: 1 \Delta 70,1-16: 2 \Delta 7,100,1-20: 1 \Delta 130,6-20: 2 \Delta 11,140,9-22: 1 \Delta 150,5-22: 2 \Delta 13,150,7-24: 1 \Delta 158,3$.

(b): $W(16,50) 0,1-X(18,77) 2,1-Y(19,79) 0,1-Z(19,85) 0,8-24: 00,2$.

(c): $L(15,07) 0,1-M(15,78) 0,2-16: 1 \Delta 70,5-N(16,62) 0,1-16: 2 \Delta 7,100,4-O(16,94) 0,1-P(17,42) 0,1-16: 3 \Delta 7,10,131,2-Q$ $(17,71) 0,1-R(17,84) 0,6-18: 2 \Delta 11,140,6$ - S (19,53) 0,2 - T (19,74) 0,1 - U (19,81) 0,5 - 20:1 $\Delta 50,4-V(20,41) 0,1-W(20,80)$ $0,3-X(21,50) 0,2-Y(21,77) 0,2-Z(23,86) 0,2$.

(d): $20: 1 \Delta 50,2-20: 2 \Delta 11,140,1$.

(e): $16: 2 \Delta 9,120,2$.

(f): $16: 1 \Delta 70,2-16: 2 \Delta 7,100,2-16: 2 \Delta 9,120,2-18: 2 \Delta 11,140,2-24: 00,2$

(g): $Y(17,84) 0,7-18: 2 \Delta 11,141,8-Z(21,50) 0,8-22: 00,8$ - 24:0 0,6.

(h): $12: 0 \quad 3,5-16: 1 \Delta 70,2$.

En la zona de LEC comprendida entre 18,00 y 20,00 se encuentran tres picos desconocidos en el caso de A.tomentosum (3\%) y tres en el caso de A.hortensis (0,8\%). EI pico de LEC 18,77 en el caso de A.tomentosum podría ser el octadecatriéno -3(t), 9(c), 12(c)- oato de metilo, habiendo sido indicado el ácido correspondiente por MORRIS et al. (Morris, 1968 ), en Arctium minus, lo que justificaría la banda IR de $972 \mathrm{~cm}^{-1}$.

En el ámbito de las LEC > 20,00 se anotan algunos compuestos poco frecuentes : $20: 1 \Delta 13(0,6 \%)$ y $22: 1 \Delta 15$ (0,5\%) en A.petiolata, $20: 1 \Delta 5$ en A.hortensis $(0,4 \%)$ y en $H$.perforatum $(0,2 \%)$. Además un cierto número de picos no identificados se contabilizan en A.hortensis (cinco por $1,0 \%$ ) y P.dysenterica (uno por $0,8 \%$ ).

Cuatro especies de las once presentadas aquí, han sido estudiadas por otros autores por su composición en ácidos grasos. Estas son A.petiolata, M.jalapa, R.rubiginosa y S.marianum. Los resultados comparados para los ácidos grasos más frecuentes están reunidos en la TABLA IV. La composición en ácidos grasos de A.petiolata es prácticamente la misma, que la especie proceda de Alemania (Hondelmann, 1984) o de la región mediterránea.

Se puede hacer la misma comprobación en el caso de R.rubiginosa (Rodríguez, 1987): las diferencias entre las poblaciones francesas y las chilenas -en cuanto a los ácidos grasos- están dentro de las desviaciones inevitables (método de análisis, condiciones ambientales, madurez de las semillas).

En cambio encontramos más divergencias entre nuestros valores y los de la literatura en el caso de M.jalapa (Patel, 1985) y S.marianum (Marquard, 1982). Eso concierne sobre todo a los ácidos oléico y linoléico. M.jalapa descrito en la literatura es originario de la India y S.marianum de Turquía. Las diferencias climáticas no pueden, solas, ser responsables de las diferencias observadas, pero no tenemos otro argumento a exponer. 
Tabla IV

Comparación entre las composiciones de ácidos grasos encontradas y las descritas en la literatura.

\begin{tabular}{|c|c|c|c|c|c|c|c|c|}
\hline \multirow{2}{*}{$\begin{array}{l}\text { aceites } \\
\text { y ácidos } \\
\text { grasos }\end{array}$} & \multicolumn{2}{|c|}{ A. petiolata } & \multicolumn{2}{|c|}{ M. jalapa } & \multicolumn{2}{|c|}{ R. rubiginosa } & \multicolumn{2}{|c|}{ S. marianum } \\
\hline & $\begin{array}{l}\text { este } \\
\text { trab. }\end{array}$ & $\begin{array}{l}\text { ref. } \\
\text { (c) }\end{array}$ & $\begin{array}{l}\text { este } \\
\text { trab. }\end{array}$ & $\begin{array}{l}\text { ref. } \\
\text { (d) }\end{array}$ & $\begin{array}{l}\text { este } \\
\text { trab. }\end{array}$ & $\begin{array}{l}\text { ref. } \\
\text { (e) }\end{array}$ & $\begin{array}{l}\text { este } \\
\text { trab. }\end{array}$ & $\begin{array}{c}\text { ref. } \\
\text { (f) }\end{array}$ \\
\hline aceite $\%$ & 15,4 & - & 4,2 & 3,0 & 9,5 & 8,0 & 24,2 & $21-28$ \\
\hline palmítico & 3,2 & 3,5 & 19,1 & 18,3 & 4,3 & 3,2 & 8,6 & 8-10 \\
\hline esteárico & 0,3 & - & 1,9 & - & 1,8 & 0,8 & 4,8 & $2-4$ \\
\hline oléico & 10,1 & 9,0 & 43,5 & 55,3 & 14,2 & 15,9 & 23,0 & $13-18$ \\
\hline linoléico & 22,5 & 24,8 & 13,1 & 11,5 & 44,3 & 40,7 & 57,1 & $70-76$ \\
\hline linolénico & 3,9 & 7,2 & 18,3 & 14,9 & 33,3 & 39,4 & 0,3 & - \\
\hline aráquico & 0,2 & - & 0,6 & - & 0,6 & - & 3,0 & - \\
\hline gondoico & 6,5 & 5,2 & 0,1 & - & 0,3 & - & 0,9 & - \\
\hline erúcico & 41,4 & 44,2 & 0,1 & - & 0,0 & - & 0,0 & - \\
\hline nervónico & 8,3 & 6,1 & 0,0 & & 0,0 & - & 0,0 & - \\
\hline
\end{tabular}

(c): Hondelmann et al., 1984.

(e): Rodríguez et al., 1987. (d): Patel et al., 1985.

(f): Marquard et al., 1982

\section{CONCLUSION}

Las especies estudiadas respecto de sus aceites de semillas en este artículo, pertenecen a tres conjuntos florísticos del Mediterráneo: las especies mediterráneas, las eurosiberianas y las naturalizadas. No hay relación evidente entre los lípidos y los ambientes, al menos en el área geográfica que nos interesa. En cambio encontramos una variedad en la naturaleza y la proporción relativa de ácidos grasos, muy interesante, a pesar de cantidades de aceite a menudo pequeñas. A grandes rasgos, los caracteres familiares se vuelven a encontrar aquí: ácido erúcico en las Brassicaceae, ácido linoléico en las Asteraceae. Estas últimas tienen a menudo ácidos grasos poco frecuentes, cosa que se verifica también aquí. La complejidad máxima se halla en Atriplex hortensis, una Chenopodiaceae, que tiene treinta y tres ácidos grasos, no todos identificados.

En conclusión, la riqueza de las observaciones en tan pequeño número de especies, nos incita a proseguir las investigaciones sobre los lípidos de la flora mediterránea.

\section{AGRADECIMIENTOS}

Agradecemos encarecidamente al Profesor J. Artaud del IUT de Chimie de Marseille, el habernos proporcionado las semillas de A.tomentosum y de H.perforatum, y al Profesor P. Gamisans de la Universidad de Toulouse, el haber repasado la traducción al castellano.

\section{BIBLIOGRAFIA}

Cerrati, C; Mallet, J.F; Ucciani ,E; Gamisans, J. y Gruber, M. (1992).- "Alfatocoferolo, un importante antiossidante nelle piante mediterranee".Riv.Ital.Sostanze Grasse 69, 317-320.

Chevolleau, S; Debal, A. y Ucciani, E. (1992).- "Détermination de l'activité antioxydante d'extraits végétaux".- Rev.Fr. Corps Gras 39, 3-8.
Chevolleau, S; Mallet, J.F; Ucciani, E; Gamisans, J. y Gruber, M. (1992)."Antioxidant activity in leaves of some mediterranean plants".- J.Am. Oil Chemists'Soc. 69, 1269-1271.

Ferlay, V; Mallet, G; Masson, A; Ucciani, E. y Gruber, M. (1993)."Composition en acides gras des huiles de graines d'espéces spontanées du sud-est méditerranéen".- Oléagineux 48, 91-97.

Fioriti, J.A. y Sims, R.J. (1968).- "The reaction of picric acid with epoxides".J.Am. Oil Chemists'Soc. 43, 487-490.

Hamilton, S. y Hamilton, R.J. (1972).-"Plant Waxes" in "Topics in Lipid Chemistry".- Vol.3, pp. 200-269,- F. D. Gunstone ed., Elek Science, London.

Hondelmann, W. y Gruner,S. (1984).- "Zur Fettsaürezüsammensetzung des Knoblauchhederichs (Alliaria petiolata (M.B) Cav. \& Gr.)".- Fette, Seifen, Anstrichm. 86, 284-286.

IUPAC (1979).- "Methodes d'analyse des matières grasses et dérivés".6 ème édition, 1ére partie, ETICG, Paris.

Madrigal, R.V. y Smith, C.R. (1982).- "Estolide triglycerides of Trewia nudiflora seed oil".- Lipids 17, 650-655.

Mallet, G. (1987).- "Methodologie de l'analyse structurale des acides gras insaturés via leurs adduits triméthyl- silyloxy".- Thése de Doctorat èsSciences, Université d'Aix-Marseille III.

Mallet, G; Dimitriades, C. y Ucciani, E. (1988).- "Quelques exemples de répartition des acides palmitoléique et cis-vaccénique entre pulpe et graine".- Rev. Fr. Corps Gras 35, 479-483.

Marquard, R. y Vömel, A. (1982).- "Gehalte und Zusammensetzung der Samenlipide von Silybum marianum (L.) Gaertn. in Abhängigkeit vom Standort sowie der Aussaat und Erntezeit".- Fette, Seifen, Anstrichm. 84, 54-59.

Mikolajczak, K.L. (1977).- "Cyanolipids".- Prog. Chem. Fats other Lipids 15, 97-130.

Morris, L. J ; Marshall, M. O. y Hammond, E. W. (1968).- "The trans-3-enoic acids of Aster alpinus and Arctium minus seed oil".- Lipids 3, 91-95.

Patel, R. G. y Patel, V. S.(1985).- "Studies on Mirabilis jalapa (four o'clock plant) seed oil".- Fette, Seife, Anstrichm. 87, 7-9.

Peyronel, D; Artaud, J; latrides, M.C; Rancurel, P; y Chevalier, J.L. (1984)."Fatty acid and squalene composition of mediterranean Centrophorus SPP egg and liver oils in relation to age".- Lipids 19, 643-648.

Rodríguez, A; Soto, G. y Valladares, J. (1987).- "Caracterización del aceite crudo de semilla de Mosqueta (Rosa aff. rubiginosa L.)".- Grasas y Aceites 38, 20-22.

Tranchant, J. (1982).- "Analyse qualitative" in "Manuel pratique de chromatographie gazeuse".- Chapitre VI, pp. 301-337, Masson, Paris.

Wolff, J. P. (1968).- "Manuel d'analyse des corps gras".- p.154, Azoulay Ed., Paris.

(Recibido: Abril 1993) 